\title{
Evaluation of menogaril in renal cell carcinoma
}

\section{A Southwest Oncology Group phase II study (8504)}

Ronald L. Stephens ${ }^{1}$, Phyllis Goodman ${ }^{2}$, E. David Crawford ${ }^{3}$, Connie F. Spicer ${ }^{1}$, Bruce A. Lowe ${ }^{4}$, Frederick R. Ahmann ${ }^{5}$, Robert Chapman ${ }^{6}$ and Ronald B. Natale

${ }^{I}$ University of Kansas Medical Center; ${ }^{2}$ Southwest Oncology Group Statistical Center; ${ }^{3}$ University of Colorado; ${ }^{4}$ Oregon Health Sciences University; ${ }^{5}$ University of Arizona; ${ }^{6}$ Henry Ford Hospital; ${ }^{7}$ University of Michigan Medical Center.

Key words: menogaril, renal cell carcinoma

\section{Summary}

The Southwest Oncology Group (SWOG) studied the response rate and toxicity of menogaril (200 $\mathrm{mg} / \mathrm{m}^{2}$ i.v. q 28 days) in patients with advanced metastatic renal cell carcinoma. During the early stage of the trial two partial responses were seen in the first 20 treated patients, and an additional 36 evaluable patients were studied. Three of $56(5 \%)$ evaluable patients achieved partial responses. Significant white cell toxicity was observed. Mild or moderate degrees of thrombocytopenia, gastrointestinal side effects, alopecia and phlebitis occurred. No cardiac toxicity was noted. The low response rate suggests that menogaril in this dose schedule has no role in the treatment of patients with advanced metastatic renal cancer.

\section{Introduction}

Menogaril is an anthracycline antibiotic synthesized from nogalamycin, and is known by the following synonyms; menogarol, 7-(12)-0-methylnogarol and 7-OMEN. Although an anthracycline its cytotoxicity may differ significantly from that of doxorubicin, as menogaril causes cell death with only minimal DNA binding $[1,2]$. Menogaril has demonstrated antineoplastic activity in several animal tumor systems; including P388 leukemia, L1210 leukemia, colon 26, colon 38, 1316 melano$\mathrm{ma}$, and $\mathrm{CD} 8 \mathrm{~F}_{1}$ mammary tumor [3]. In a direct comparison of menogaril and doxorubicin the two agents were found to be equally effective against i.p. P388 leukemia, menogaril was more active against i.v. inoculated P388 and i.p. inoculated L1210, but less active than doxorubicin against i.p. inoculated B16 melanoma [4].

Two separate phase I trials have been published, and both studies recommended that the starting dose of menogaril in previously untreated patients be $200 \mathrm{mg} / \mathrm{m}^{2}[5,6]$. Neither of these phase I trials noted responses in the patients studied. Dose limiting toxicity was primarily myelosuppression, although also reported were local phlebitis and/or erythema, infrequent alopecia, and minor arrhythmias. The Southwest Oncology Group (SWOG) studied menogaril in several different diseases, and this report details the phase II results seen in patients with renal cancer. Study objectives were twofold, first to determine the response rate and second to assess the toxicities of menogaril administered every 28 days.

\section{Materials and methods}

Eligibility included a histologic confirmation of renal cell carcinoma, no prior chemotherapy, bi- 
dimensionally measurable disease, life expectancy of six weeks, performance status of 3 or better (SWOG criteria), and an absence of a recent myocardial infarction (prior six months) or history of congestive heart failure or cardiac arrhythmia. $\mathrm{Pa}$ tients were to have recovered from prior surgery and radiation. Concomitant radiation was allowed to lesions not being measured for purposes of the study, and could not exceed a bone marrow volume of $10 \%$. Concomitant hormonal therapy or chemotherapy were not allowed. Laboratory criteria for eligibility included a granulocyte count greater than or equal to $2000 / \mathrm{ul}$, platelet count greater than or equal to $100,000 / \mathrm{ul}$, bilirubin less than $2.0 \mathrm{mg} \%$, SGOT less than $1.5 \times$ normal, and a creatinine of less than $2.0 \mathrm{mg} \%$. All patients were provided information as to the investigational nature of the study and were required to sign a written document of informed consent in keeping with institutional and FDA guidelines.

All patients received an initial dose of menogaril at $200 \mathrm{mg} / \mathrm{m}^{2}$ i.v. over one hour in $500 \mathrm{cc}$ of $5 \%$ dextrose in water. Subsequent doses were administered at 28 day intervals and increased by $20 \%$ if the nadir granulocyte count was greater than $1500 / \mathrm{ul}$ and platelet nadir exceeded $100,000 / \mathrm{ul}$. For nadir granulocyte counts between $1000-1499 /$ ul or platelet counts between 50,000-99,999/ul, the dose remained the same. For nadir granulocyte counts lower than $1000 /$ ul or platelet counts less than $50,000 / \mathrm{ul}$, the subsequent dose of menogaril was decreased by $20 \%$.

Study patients underwent history and physical examination every 28 days, tumor measurements at this same interval, weekly blood counts, and chemistries every 28 days. Response criteria were standard for a phase II trial: including a complete remission (CR) which required disappearance of all clinically detectable disease for at least four weeks, a partial remission (PR) which required a $50 \%$ shrinkage in the sum of the products of all diameters of measured lesions for a minimum of four weeks, $30 \%$ reduction in the sums of liver measurements below the costal margins, partial calcification of lytic bone lesions or $50 \%$ reduction in blastic lesions, and no simultaneous increase in the size of any lesion or the appearance of new lesions. Pro- gression was defined as at least a $25 \%$ increase in the size of any measurable lesion, appearance of a new lesion, uncontrolled hypercalcemia, or clear progression of lytic bone lesions. Relapse was defined as the appearance of new lesions, reappearance of old lesions in patients with a previous $\mathrm{CR}$, or $50 \%$ increase over the sum of the products of diameters noted at maximal PR.

For statistical purposes this study was designed for two stages. The first stage was to enroll 20 eligible patients and temporarily close the trial for response determination. If no responses or remissions were seen the trial would be closed as negative. If any remissions were seen the second stage was designed to enter an additional 20 eligible patients and remission rate reevaluated. If four or fewer remissions (CR and PR) were seen the drug would be rejected as inactive for further study at this schedule in patients with renal cancer.

\section{Results}

Thirty-one of 56 patients $(55 \%)$ had undergone nephrectomy, 9/56 (16\%) had received some form of prior immunotherapy, and 11/56 (20\%) had experienced prior hormone treatment. Except for 11 patients all of those entered on the trial received two or more cycles of chemotherapy. Of the 11 patients receiving less than two cycles of therapy, seven were clearly progressing before the 28 day cycle could be repeated, two experienced early death, and two patients left the care of the participating investigator before receiving a second dose.

After the first 20 patients were registered two PR's were seen, and the trial was reopened to assure a more adequate determination of response. Ultimately a total of 57 patients were studied, 56 of whom were evaluable for response and toxicity. One patient was ineligible due to a diagnosis of prostate cancer, and was inadvertently registered on this study instead of the simultaneously ongoing SWOG menogaril trial in this disease. Three PR's were seen. One patient had a primary renal lesion which responded for seven months, one patient had a PR in a subcutaneous nodule which lasted for two months, and the 3 rd patient with a PR had a re- 
Table 1. Toxicities

\begin{tabular}{|c|c|c|c|c|c|c|}
\hline \multicolumn{7}{|l|}{ TOXICITIES } \\
\hline & Grade & None & Mild & Mod & Severe & LT \\
\hline WBC & & 9 & 11 & 19 & 14 & 3 \\
\hline Platelet & & 52 & 0 & 4 & 0 & 0 \\
\hline GI & & 33 & 10 & 7 & 1 & 0 \\
\hline Granulocytopenia & & 15 & 3 & 11 & 18 & 9 \\
\hline \multicolumn{7}{|c|}{ OTHER TOXICITY } \\
\hline & Grade & None & Mild & Mod & Severe & LT \\
\hline Anemia & & 30 & 10 & 10 & 6 & 0 \\
\hline Alopecia & & 41 & 7 & 5 & 3 & 0 \\
\hline Phlebitis & & 51 & 1 & 3 & 1 & 0 \\
\hline Anorexia & & 52 & 2 & 1 & 0 & 1 \\
\hline Fatigue & & 53 & 2 & 1 & 0 & 0 \\
\hline Allergic reaction & & 55 & 0 & 1 & 0 & 0 \\
\hline
\end{tabular}

sponding lung lesion of five and one-half months. No CR's were seen, and the overall response rate was five percent, with an exact $95 \%$ confidence interval of $1 \%$ to $15 \%$.

A median of two courses of menogaril (range $1-11$ courses) was given for the 56 evaluable patients. The amount of menogaril administered per patient ranged from $190.5 \mathrm{mg} / \mathrm{m}^{2}$ to $1600 \mathrm{mg} / \mathrm{m}^{2}$ with a median dose of $630 \mathrm{mg} / \mathrm{m}^{2}$. There were 17 dose escalations and 36 dose reductions given per protocol criteria.

Toxicity is summarized in Table 1. Toxicity grading is by standard SWOG criteria. The major side effect in this patient population was in the white cells, where $17 / 56(30 \%)$ patients sustained severe or life-threatening toxicity. Significant thrombocytopenia was rarely seen, and gastrointestinal toxicity was generally mild or absent. Other toxicities included anemia $(46 \%)$, alopecia $(27 \%)$ and infrequent phlebitis $(9 \%)$. No instances of acute or chronic cardiac toxicity were observed.

\section{Discussion}

Patients with advanced metastatic renal cell carcinoma remain relatively resistant to any form of systemic therapy. This dose schedule of menogaril was relatively well tolerated, although significant white cell depression did occur. This compound appears to have less gastrointestinal toxicity than doxorubi- cin. That menogaril demonstrated no long term cardiac toxicity may be related to the lower dose and shorter exposure in patients with resistant renal cell carcinoma. There are no known anthracycline chemotherapeutic agents that have any efficacy in renal carcinoma. The finding of only three responders out of $56(5 \%)$ strongly suggests that menogaril in this dose schedule also has no role in the treatment of patients with advanced renal cell carcinoma.

\section{Acknowledgements}

This investigation was supported in part by the following PHS Cooperative Agreement grant numbers awarded by the National Cancer Institute, DHHS: CA-12644, CA-37429, CA-12213, CA13612, CA-04915, CA-27057, CA-32734, CA04919, CA-16385, CA-03096, CA-04920, CA35995, CA-21116, CA-37981, CA-22411, CA20319, CA-28862, CA-14028, CA-32102.

\section{References}

1. Kruger WC, Pschigoda LM, Schpok SLF, Moscowitz A, McGovren JI, Neta P, Merritt MV, Li LH: The interaction of nogalamycin and analogs with DNA and other biopolymers. Chem-Biol. Interaction 36: 1-18, 1981

2. Li LH, Murch LL, Wooden JM, Krueger WC, Pschigoda LM: Inhibition of DNA and RNA polymerases by nogalamycin and its analogs. (Abstr) Proc Am Assoc Cancer Res 19: 30, 1978

3. Neil GL, Kuentzel SL, McGovren JP: Treatment of mouse tumors with 7-con-0-Methylnogarol and other analogs of the anthracycline antibiotics, nogalamycin. Cancer Treat Rep 63: 1971-1978, 1979

4. Bhuryan BK, McGovren JP, Crampton SL: Intracellular uptake of 7-con-0-Methylnogarol and Adriamycin by cells in culture and its relationship to cell survival. Cancer Res 41: $882-887,1981$

5. Dorr FA, VonHoff DD, Kuhn JG, Schwartz R, Krisner DL: Phase I clinical investigation of 7-con-0-Methylnogaril, a new anthracycline antibiotic. Cancer Res 46: 2562-2565, 1986

6. Dodion P, Sessa C, Joss R, Crespeigne N, Willems Y, Kitt M, Abrams J, Finet C, Brewer JE, Adams WJ, Earhart RH, Rozencweig M, Kenis Y, Cavalli F: Phase I study of intravenous menogaril administered intermittently. J Clin Oncol 4: 767-774, 1986

Address for offprints: Southwest Oncology Group (SWOG8504), Operations Office, 5430 Fredericksburg Road, Suite \#618, San Antonio, TX 78229, USA 